\title{
Removing shadow for hand segmentation based on background subtraction
}

\begin{abstract}
Hand segmentation is an important stage for accurate hand detection and background subtraction is one of the best solutions to detect the hand motion accurately, however the shadow is the critical problem in this technique which is not easy to separate the hand region from the shadow area. Removing shadow using an automatic threshold will be a good solution to detect the hand region where the variety of skin color and lighting condition affect the hand segmentation. The proposed approach involves three stages: First, we convert RGB color model to YUV space to get the benefit of separation the luminance channel (Y) from the chrominance channels ( $U, V)$ to reduce the effect of shadow, reflections and, etc. In the second stage, we applied background subtraction technique to the $\mathrm{V}$ channel to remove the unwanted background noise and to get the hand and shadow pixels. Finally, we used shareholding technique by considering a mean value of the pixels of foreground image (the hand and shadow pixels) as automatic threshold value and other tow static thresholds to distinguish the hand region from shadow pixels. After background subtraction, we used the famous morphology techniques (Erosion and Dilation) to enhance the accuracy of hand detection. We measure the accuracy for the results by compare the detect hand pixels to the actual hand pixels quantitatively. From the results, we noticed that our proposed approach is accurate and suitable for real time application systems.
\end{abstract}

Keyword: Background subtraction; Automatic thresholding; Hand segmentation; Removing shadow 